\title{
The Accuracy of Identifying the Greater Palatine Neurovascular Bundle: A Cadaver Study
}

\author{
Jia-Hui Fu, * Dawlat G. Hasso, * Chu-Yuan Yeh, * Daylene J.M. Leong, * Hsun-Liang Chan, *
} and Hom-Lay Wang*

Background: The palate is a common site for harvesting subepithelial connective tissue grafts (SCTG). The size of SCTG that can be harvested is dictated by the position of the greater palatine neurovascular bundle (GPB). The aims of this cadaver study are to assess the accuracy of predicting the location of the GPB on study models and to evaluate anatomic factors that might influence the predictability.

Methods: Eleven fully dentate or partially edentulous maxillary cadavers were used. Study models were fabricated after the greater palatine foramen was identified. The GPB was recognized after dissection, from which the distance to the cemento-enamel junction of the first molar and premolar was measured. Eight periodontists and twelve periodontal residents were asked to estimate the location of the GPB on the study models and the same measurements were taken. Comparisons of the estimated and true GPB position were performed. The correlation between the palatal vault height and the variability of detecting the GPB was investigated.

Results: The most frequent greater palatine foramen location was between the second and third molars (66.6\%). For most cases, there was an underestimation of the location of the GPB up to $4 \mathrm{~mm}$. The interexaminer variability was positively correlated with the vault height.

Conclusions: The estimated location of the GPB was commonly closer to the cemento-enamel junction of posterior teeth. Agreement on the location of the GPB was lowered with the presence of high palatal vaults. The results of this study could assist clinicians in planning the location for harvesting SCTG on the hard palate. J Periodontol 2011;82:1000-1006.

\section{KEY WORDS}

Anatomy; connective tissue; gingival recession; maxillary nerve; palate surgery, plastic.

\footnotetext{
* Graduate Periodontics, School of Dentistry, University of Michigan, Ann Arbor, MI.
}

$M$ ucogingival deformity is defined as a departure from the normal dimension and morphology of or interrelationship between gingiva and alveolar mucosa, which may be associated with a defect in the underlying alveolar bone. ${ }^{1}$ One common example of a mucogingival deformity is gingival recession, which is the movement of the gingival margin apical to the cementoenamel junction (CEJ). ${ }^{1}$ The prevalence of gingival recession ranges from $0 \%$ to $100 \%$ depending on the population, age, anatomic factors, physiologic factors, pathologic factors, trauma, and level of oral hygiene. ${ }^{2-10}$ In a population of Tanzanian women, $33.6 \%$ had gingival recession of $\geq 1 \mathrm{~mm} .{ }^{4}$ Toker and Ozdemir $^{5}$ found that only $17.4 \%$ had gingival recession $<2 \mathrm{~mm}$. A longitudinal study, involving a well-maintained and dentally aware Norwegian population and a Sri Lankan population who had no access to dental care, observed that the prevalence of gingival recession was similar, occurring in $60 \%$ to $90 \%$ and $30 \%$ to $100 \%$ of the subjects, respectively, although the etiology of gingival recession of the two distinct groups may have differed. ${ }^{2}$ Hugoson et al. ${ }^{3}$ examined a large random Swedish population and found that the frequency of tooth surfaces with gingival recession increased from $0 \%$ in 20 -year-old subjects to $22.2 \%$ in 80-year-old subjects. This concurs with the observation from other studies 
that the prevalence of gingival recession increases with age. 9,10

Several classification systems were proposed to better describe gingival recession defects. In 1968, Sullivan and Atkins ${ }^{11}$ provided a descriptive categorization of gingival recession defects into the combinations of narrow, wide, shallow, and deep groups. Miller, ${ }^{12}$ based on the level of interproximal soft and hard tissues and the relationship of the facial gingival margin to the mucogingival junction, proposed four classes of gingival recession. Recently, Nordland and Tarnow ${ }^{13}$ formulated a classification system that examined the papillary fill and its relation to the CEJ. Of these classification systems, Miller's classification is most commonly used because it not only provided the expected clinical success rate for root coverage but also emphasized the importance of interproximal bone support in ensuring treatment success.

Root coverage procedures can be broadly categorized into pedicle grafts and free soft-tissue grafts. Pedicle grafts, such as laterally positioned flap, ${ }^{14}$ double-pedicle flap, ${ }^{15}$ oblique rotational flap, ${ }^{16}$ and coronally positioned flap, ${ }^{17}$ have a key advantage over free soft-tissue grafts in that the blood supply to the flap is maintained. Unfortunately, there is limited increase in tissue thickness and width of keratinized gingiva with the use of these surgical techniques. Free soft-tissue grafts, such as free gingival graft ${ }^{18}$ and subepithelial connective tissue graft (SCTG), 19 however, are able to increase the width of keratinized gingiva and tissue thickness. Autogenous tissue grafts do possess several drawbacks, such as donor site morbidity ${ }^{20}$ and limited tissue availability. ${ }^{21}$ As such, allogenic tissue grafts, such as acellular dermal matrix $^{22,23}$ and bilayered cell therapy, 24,25 were developed to serve as alternatives to autogenous grafts with favorable treatment outcomes. ${ }^{24,26}$ Based on currently available literature, SCTG in combination with a coronally positioned flap was statistically superior in achieving root coverage compared to free gingival graft, coronally positioned flap alone, guided tissue regeneration, and allogenic tissue grafts. ${ }^{27-30}$

An SCTG is commonly harvested from the hard palate. ${ }^{31,32}$ According to Langer and Langer, ${ }^{33}$ a horizontal incision to bone is made $5 \mathrm{~mm}$ from the palatal gingival margin and the blade is subsequently placed parallel to the long axis of the roots. Another horizontal incision is made $2 \mathrm{~mm}$ coronal to the first incision and the periosteum is dissected before removing the wedge of soft tissue. Great care should be taken during the harvesting procedure to avoid violating the greater palatine neurovascular bundle (GPB). ${ }^{34}$ The GPB, which contains the greater palatine artery, vein, and nerve, travels in the pterygopalatine fossa, passes through the pterygopalatine canal, and exits through the greater palatine foramen (GPF). ${ }^{35}$
After entering the oral cavity, it runs anteriorly in a bony groove between the junction of the maxillary alveolar ridge and the horizontal plate of the maxilla and anastomoses in the premaxilla with branches of the nasopalatine bundle from the nasal cavity. ${ }^{32}$

The amount of SCTG that can be harvested is dictated by the thickness of the palatal mucosa and the location of the GPB. The graft is generally taken anterior to the mesial line angle of the first molar and posterior to the canine. This is because the palatal tissue thickness is minimal at the first molar, on top of which, the GPB branches and courses more coronally beyond the canine region. ${ }^{32}$ The location of the GPB, in relation to the height of the palatal vault, was found to be on average 7,12 , and $17 \mathrm{~mm}$ from the CEJs of the premolars and molars in shallow, average, and high palatal vaults, respectively. ${ }^{32}$ Based on the aforementioned anatomic landmarks, the maximum graft dimensions of $8 \mathrm{~mm}$ in height and $31.7 \mathrm{~mm}$ in length were demonstrated on stone casts of periodontally healthy subjects. ${ }^{36}$ The emergence of the GPB was assumed to be localized at the junction of the vertical and horizontal palatal walls of the vault. The maximum height of the graft corresponded to the distance between the gingival margin and the assumed course of the GPB. However, the accuracy of locating the course of the GPB on stone models was not validated. Valuable clinical information could be obtained if discrepancy between the estimated location of the GPB on study casts and the true bundle location could be determined. Therefore, this study on cadaveric specimens aims to assess the accuracy of predicting the location and course of the GPB on study models, to compare it to the anatomic findings, and also to evaluate anatomic factors that might influence the predictability.

\section{MATERIALS AND METHODS}

Eleven fresh cadaver heads with fully dentate or partially edentulous maxilla were used in this study. The cadavers were donated to the Department of Periodontics and Oral Medicine, University of Michigan School of Dentistry, Ann Arbor, Michigan, for educational purposes. The specimens belonged to white male subjects with an age range of 58 to 91 years (mean age: 75.7 years).

\section{GPB Measurements on Cadavers}

The GPF was located using a 22-gauge hypodermic needle. ${ }^{\dagger}$ The palatal gingiva of posterior teeth was excised with a \#15 blade b $^{\ddagger}$ to expose the CEJ. Each maxillary arch was replicated with vinyl polysiloxane impression material.§ Gypsum model stonel| was used

\footnotetext{
$\dagger$ Becton Dickinson, Franklin Lakes, NJ.

\# Disposable Blade \#15, Becton Dickinson.

$\S$ Aquasil impression material, DENSTPLY International, York, PA

\| Microstone, Whip Mix Corporation, Louisville, KY.
} 
to fabricate study models with hypodermic needles in the GPF. Manufacturer instructions were followed during the acquisition of the impressions and study models.

The GPB was exposed with mask dissection beginning from the GPF to the canine region. The GPB location was determined by measuring its vertical distance from the CEJs of the first molar (BMV) and first premolar (BPV) (Fig. 1). A periodontal probe was positioned parallel to the palatal vault at the CEJ of a reference tooth. A stainless steel ruler," accurate to $1 \mathrm{~mm}$, was then placed on palatal mucosa overlying the GPB and perpendicular to the probe. The measurement was taken at the point where the probe and ruler intersected. In specimens where a reference tooth was missing, the next tooth of the same type was used. The vertical distance of the GPF was measured with the same method. GPF location in relation to the tooth was also determined. Three examiners (DGH, C-YY, and DJML) performed the dissections and measurements.

\section{GPB Prediction and Measurements on Stone Models}

Eight periodontists and 12 residents from the Graduate Periodontics Department at the School of Dentistry, University of Michigan, participated in this study. A piece of translucent tape** was fixed onto the palate of the study models before it was delivered to each participant. Participants were asked to draw the estimated location of the GPB on the models with

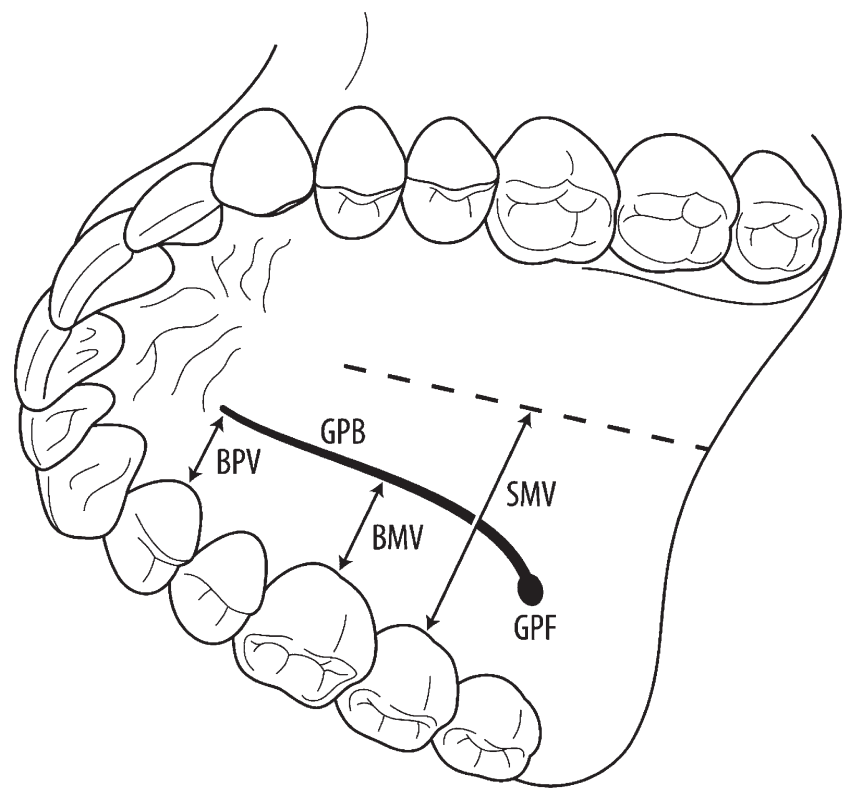

Figure 1 .

Depiction of the relationship between the GPB and maxillary posterior teeth and relevant measurements. The broken line indicates the midsagittal palate. SMV = vault height. a fine-tip red pen. ${ }^{\dagger \dagger}$ The same measurements on the casts (BMVc and BPVc) were obtained by a single examiner (DGH) with the method as used in the cadaveric specimens. Once all measurements were finished for one participant, the translucent tape was removed and a new piece of tape was adapted on the palate. The models were transferred to the next participant until all measurements were obtained from all participants. In addition, the vault height (SMV) of all 11 specimens, defined as the vertical distance from the CEJ of the second molar to the palate in the midsagittal plane, was measured by a single examiner (DGH).

\section{Accuracy Assessment}

For each participant, the vertical distance differences of the GPB between the cadaveric and the model measurements were calculated by subtracting the former from the latter. The equations used were as follows:

$$
\begin{aligned}
\mathrm{BMVd} & =\mathrm{BMVc}-\mathrm{BMV} \\
\mathrm{BPVd} & =\mathrm{BPV} \mathrm{C}-\mathrm{BPV}
\end{aligned}
$$

A negative value indicated an underestimation of the position of the GPB and the opposite equated to an overestimation. The mean values, BMVd and BPVd, were taken using these formulae: mean $\mathrm{BMVd}=\operatorname{sum}\left(\mathrm{BMVd}_{\mathrm{n}}=1\right.$ to 20$) / 20$ and mean $\mathrm{BPVd}=$ sum $\left(\mathrm{BPVd}_{\mathrm{n}=1 \text { to } 20}\right) / 20$. SD_BMVd and SD_BPVd represented the standard deviation of BMVd and BPVd, respectively.

\section{Statistical Analysis}

The variables, including $B M V c, B M V d, B P V c, B P V d$, SMV, and GPF, and their derivatives were presented as mean \pm SD in millimeters. The SD_BMVd and SD_BPVd in relation to the vault height (SMV) was scatter plotted and the correlation was determined with the Pearson correlation. The association was considered significant when $P<0.05$. The intraclass correlation coefficient was used to evaluate the intraexaminer and interexaminer reliability of the measurements. Intraclass correlation coefficient values equal to 0 represent agreement equivalent to that expected by chance, whereas 1 represents perfect agreement. Non-parametric independent sample test was used to investigate if a difference in experience level impacts the accuracy of identifying the GPB. Statistical analysis was processed with a commercially available software package. ${ }^{\ddagger}$ 


\section{RESULTS}

Table1 summarizes GPF, GPB position in relation to posterior teeth, and vault height in cadavers. Twenty-one greater palatine foramina were available for evaluation (one was excluded because of lack of a reference tooth). The most frequent location was between the second and third molars (66.6\%), followed by palatal to second molars $(19.1 \%)$ and palatal to third molars (14.3\%). The mean distance between GPF and CEJ of second or third molar, depending on location of GPF, was $14.5 \pm 2.4 \mathrm{~mm}$. The average palatal vault height (SMV) was $14.1 \pm 2.5 \mathrm{~mm}$. The mean vertical distance from the neurovascular bundle to the CEJ was $13.1 \pm 2 \mathrm{~mm}$ and $12.2 \pm 2 \mathrm{~mm}$ at the first molar (BMV) and first premolar site (BPV). In most instances, the mean BPVd and BMVd ranged between 0 and $-4 \mathrm{~mm}$ and 0 and $-3 \mathrm{~mm}$, respectively (Fig. 2). The standard deviation of BPVd (SD_BMVd) and that of BMVd (SD_BMVd) was positively correlated with the SMV $\left(r^{2}=0.393\right.$ and 0.586) (Fig. 3). No significant difference was found between the periodontists and residents in identifying the GPB. Intraexaminer reliability among three examiners was $0.83(P<0.001)$. Interexaminer reliability was between 0.92 and 0.95 for the three examiners $(P<0.001)$.

\section{DISCUSSION}

The GPB is probably the most important anatomic structure to consider when a connective tissue graft is harvested from the palate. The probable complications when this bundle is encroached are bleeding and paresthesia, although the incidence is low. ${ }^{20,34}$ Most often, the surgery is planned on study models. Although the bundle is known to be located at the junction of the maxillary alveolar ridge and the horizontal plate of the maxilla, the thickness of palate tissue and curvature of palatal vault might obscure its actual position and hinder the clinician from accurately locating it on the model. No studies have investigated the predictability of identifying the GPB and factors that may influence the predictability. This article served this purpose.

The most significant finding of this study is the existence of a discrepancy between the estimated GPB location on the models and the true location as determined on the cadaveric specimens. Most participants tend to underestimate the location of the GPB and the discrepancy could be up to $4 \mathrm{~mm}$. For most clinical scenarios, the underestimation might be advantageous because the chance of injuring the GPB was greatly reduced. However, if a maximal amount of connective

\section{Table I.}

\section{The GPB and GPF in Relation to the Posterior Teeth and Vault Height of Individual Cadavers ( $\mathrm{mm}$ )}

\begin{tabular}{|c|c|c|c|c|c|c|c|c|}
\hline \multirow[b]{2}{*}{ Cadaver No. } & \multicolumn{4}{|c|}{ Right Side } & \multicolumn{4}{|c|}{ Left Side } \\
\hline & SMV & BMV & BPV & GPF & SMV & BMV & BPV & GPF \\
\hline 1 & 14 & 15 & 14 & 15 & 14 & 11 & 12 & 17 \\
\hline 2 & 12 & 12 & 12 & 13 & 11 & 11 & 12 & 11 \\
\hline 3 & - & - & 12 & 15 & 12 & 11 & 12 & 12 \\
\hline 4 & 14 & 14 & 14 & 13 & 15 & 12 & 11 & 16 \\
\hline 5 & - & - & - & 14 & 19 & 17 & 16 & 20 \\
\hline 6 & - & - & 10 & 13 & - & - & 12 & - \\
\hline 7 & 14 & 11 & - & 15 & 15 & 11 & - & 17 \\
\hline 8 & - & - & 10 & 15 & - & - & 7 & 15 \\
\hline 9 & 12 & 12 & 12 & 13 & 13 & 14 & 13 & 12 \\
\hline 10 & 14 & 14 & 13 & 16 & 11 & 14 & 15 & 11 \\
\hline 11 & 19 & 15 & - & 17 & 17 & 16 & 12 & 18 \\
\hline Range & 11 to 19 & 11 to 17 & 7 to 16 & II to 20 & & & & \\
\hline Mean (SD) & |4.| (2.5) & I3.1 (2.0) & I2.2 (2.0) & | 4.5 (2.4) & & & & \\
\hline
\end{tabular}

$-=$ data not available because of missing reference teeth.

Range and mean include both the right and left sides. 

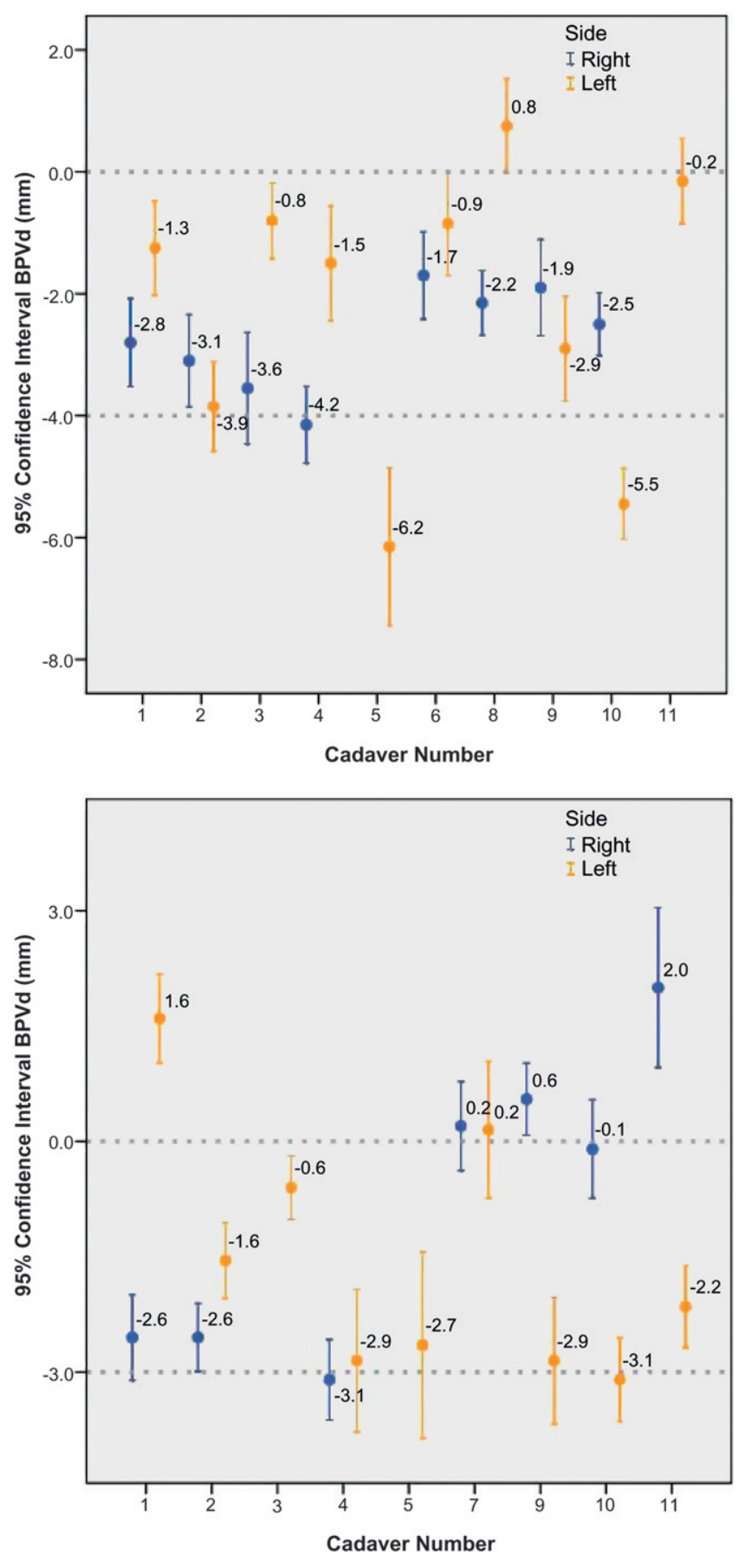

Figure 2.

Mean difference $\pm 95 \%$ confidence interval between the estimated (top) and true actual (bottom) GPB position measured on the casts and cadavers.

tissue graft had to be harvested, the underestimation might limit the size of the graft. In the current study, the participants were not allowed to palpate the palates of the cadaveric specimens. Therefore, this might have resulted in a higher incidence of the bundle being misidentified.

The variability in recognizing the GPB among participants was positively correlated with the vault height $\left(r^{2}=0.393\right.$ to 0.586 when the GPB was estimated in the premolar and molar sites, respectively). The higher the palatal vault, the lower the participant agreement on the location of the bundle. It is possible that the high palatal vault had a less distinct horizontal and vertical maxillary junction, which complicated the identification of the GPB. The clinical significance of this finding is that greater care should be given to patients with a higher vault when harvesting a connective tissue graft in the palate.

The GPF was found to be located mainly between the second and third molars (66.6\%) and this was comparable to other studies. ${ }^{31,37}$ Wang et al. ${ }^{37}$ investigated adult Chinese skulls and found that the most common location of the GPF was between the second and third molars (48\%) followed by the position palatal to the third molar (33.5\%). Klosek and Rungruang ${ }^{31}$ reported that the most frequent position of the GPF was palatal to the second molar $(35.7 \%)$ and interproximal to the second and third molars in women $(35.7 \%)$ and palatal to the second molar in men (65\%) in Thai cadaveric specimens. The present study confirmed the previously mentioned studies, demonstrating that the most common GPF location was between the second and third molars. This information could assist clinicians in identifying the GPF when performing the nerve block.

During the dissection of cadaveric specimens, the course and dimension of the GPB was observed. After leaving the GPF, the GPB traveled as a thick bundle adjacent to the molars. Its diameter decreased gradually as branching started at the level of the premolars. Similar findings were reported and the diameter of the greater palatine artery was thickest $(2.65 \pm$ $1.3 \mathrm{~mm}$ ) at the GPF, gradually decreased to $1.96 \pm$ $0.9 \mathrm{~mm}$ at the first premolar area and $1.1 \pm 0.5 \mathrm{~mm}$ at the incisive foramen. ${ }^{31}$ Branching was observed almost along the whole course of the GPA and more often toward the alveolar side than the hard palate side. ${ }^{31}$ However, the GPA had the most branches at the first premolar in women (38\%). In men, the branching on the alveolar process side was commonly observed at the first and second premolars (56\%). As a result, not only the location of the main trunk of the GPB but also that of its branches should be understood so that surgical complications can be reduced.

\section{CONCLUSIONS}

In this cadaver study, the accuracy of identifying the GPB at posterior maxillary teeth was investigated. The estimated location of the GPB as determined on the study models, compared to the true GPB position revealed after dissection, was more commonly closer to the CEJs of posterior teeth. The interexaminer variability was positively correlated with the 

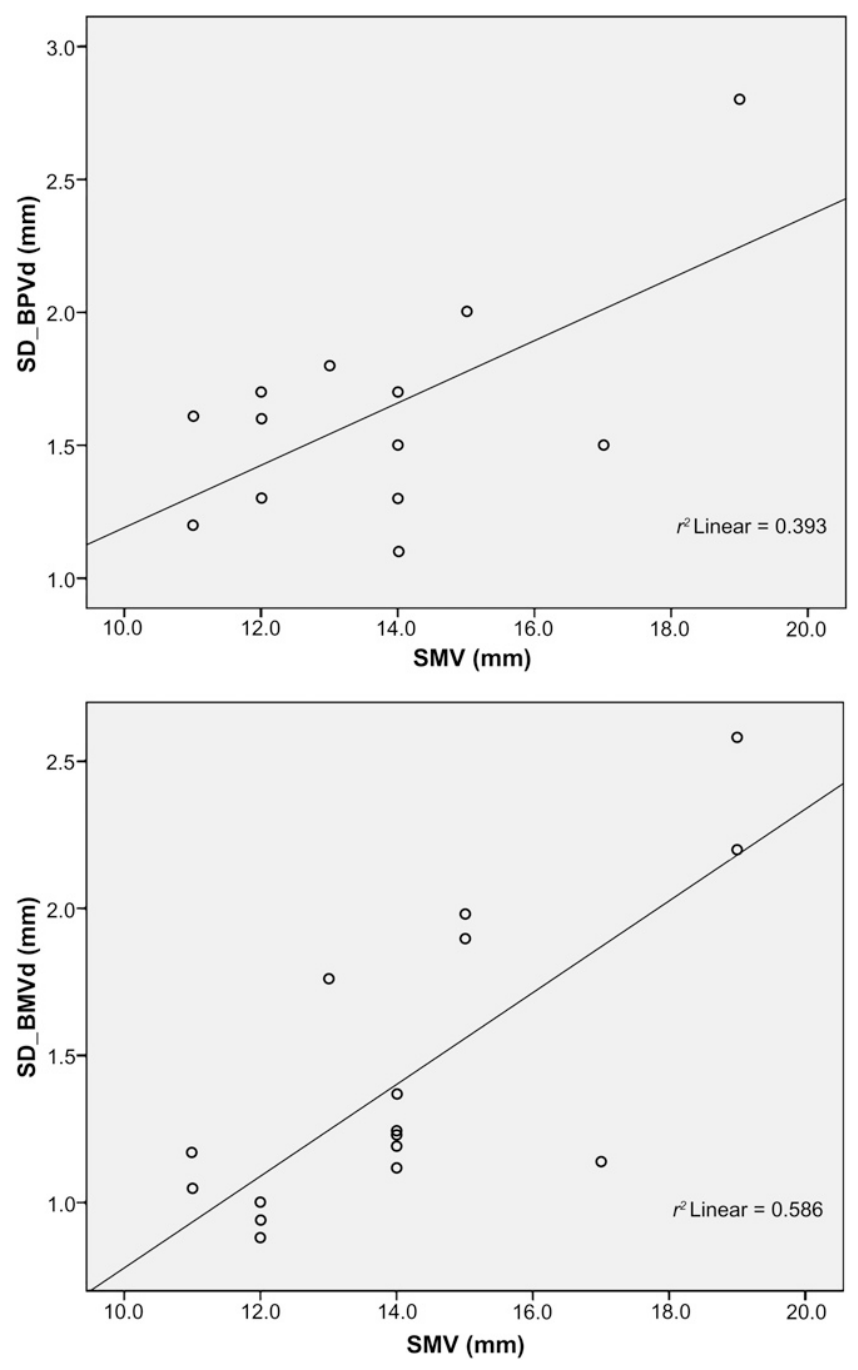

Figure 3.

Correlation between the standard deviation of the BPVd and BMVd and the vault height.

vault height. For high vault cases, agreement on the position of the GPB among participants was lower. The results of this study could assist clinicians in planning connective tissue graft procedures on the hard palate.

\section{ACKNOWLEDGMENTS}

The authors thank Dean Muller and his staff in the Anatomy Department, University of Michigan, for the organization and transportation of cadaver heads. In addition, they acknowledge the efforts of Ms. Dalia Hasso, high school student, summer externship, Detroit, Michigan, in managing the logistics of this study. This paper was partially supported by the University of Michigan Periodontal Graduate Student Research Fund. The authors report no conflicts of interest related to this study.

\section{REFERENCES}

1. American Accademy of Periodontology. Glossary of Periodontal Terms. Chicago: American Academy of Periodontology; 2001:33.

2. Löe H, Anerud A, Boysen H. The natural history of periodontal disease in man: Prevalence, severity, and extent of gingival recession. J Periodontol 1992;63: 489-495.

3. Hugoson A, Sjödin B, Norderyd O. Trends over 30 years, 1973-2003, in the prevalence and severity of periodontal disease. J Clin Periodontol 2008;35:405414.

4. Mumghamba EG, Honkala S, Honkala E, Manji KP. Gingival recession, oral hygiene and associated factors among Tanzanian women. East Afr Med J 2009; 86:125-132.

5. Toker H, Ozdemir H. Gingival recession: Epidemiology and risk indicators in a university dental hospital in Turkey. Int J Dent Hyg 2009;7:115-120.

6. Susin C, Haas AN, Oppermann RV, Haugejorden O, Albandar JM. Gingival recession: Epidemiology and risk indicators in a representative urban Brazilian population. J Periodontol 2004;75:1377-1386.

7. Kassab MM, Cohen RE. The etiology and prevalence of gingival recession. J Am Dent Assoc 2003;134:220 225.

8. Albandar JM, Kingman A. Gingival recession, gingival bleeding, and dental calculus in adults 30 years of age and older in the United States, 1988-1994. J Periodontol 1999;70:30-43.

9. Gorman WJ. Prevalence and etiology of gingival recession. J Periodontol 1967;38:316-322.

10. Murray JJ. Gingival recession in tooth types in high fluoride and low fluoride areas. $J$ Periodontal Res 1973;8:243-251.

11. Sullivan HC, Atkins JH. Free autogenous gingival grafts. 3. Utilization of grafts in the treatment of gingival recession. Periodontics 1968;6:152-160.

12. Miller PD Jr. A classification of marginal tissue recession. Int J Periodontics Restorative Dent 1985;5(2): 8-13.

13. Nordland WP, Tarnow DP. A classification system for loss of papillary height. J Periodontol 1998;69:11241126.

14. Grupe HE, Warren RF. Repair of gingival defects by a sliding flap operation. J Periodontol 1956;27:92-95.

15. Cohen DW, Ross SE. The double papillae repositioned flap in periodontal therapy. J Periodontol 1968;39: 65-70.

16. Pennel BM, Higgason JD, Towner JD, King KO, Fritz BD, Salder JF. Oblique rotated flap. J Periodontol 1965;36:305-309.

17. Harvey PM. Management of advanced periodontitis. I. Preliminary report of a method of surgical reconstruction. N Z Dent J 1965;61:180-187.

18. Nabers JM. Free gingival grafts. Periodontics 1966;4: 243-245.

19. Edel A. Clinical evaluation of free connective tissue grafts used to increase the width of keratinised gingiva. J Clin Periodontol 1974;1:185-196.

20. Griffin TJ, Cheung WS, Zavras AI, Damoulis PD. Postoperative complications following gingival augmentation procedures. J Periodontol 2006;77:20702079.

21. Soileau KM, Brannon RB. A histologic evaluation of various stages of palatal healing following subepithelial 
connective tissue grafting procedures: A comparison of eight cases. J Periodontol 2006;77:1267-1273.

22. Harris RJ. Root coverage with a connective tissue with partial thickness double pedicle graft and an acellular dermal matrix graft: A clinical and histological evaluation of a case report. J Periodontol 1998;69: 1305-1311.

23. Woodyard JG, Greenwell H, Hill M, Drisko C, Iasella JM, Scheetz J. The clinical effect of acellular dermal matrix on gingival thickness and root coverage compared to coronally positioned flap alone. J Periodontol 2004;75:44-56.

24. McGuire MK, Scheyer ET, Nunn ME, Lavin PT. A pilot study to evaluate a tissue-engineered bilayered cell therapy as an alternative to tissue from the palate. $J$ Periodontol 2008;79:1847-1856.

25. Nevins ML. Tissue-engineered bilayered cell therapy for the treatment of oral mucosal defects: A case series. Int J Periodontics Restorative Dent 2010;30:31-39.

26. Harris RJ. Soft tissue ridge augmentation with an acellular dermal matrix. Int J Periodontics Restorative Dent 2003;23:87-92.

27. Oates TW, Robinson M, Gunsolley JC. Surgical therapies for the treatment of gingival recession. A systematic review. Ann Periodontol 2003;8:303-320.

28. Roccuzzo M, Bunino M, Needleman I, Sanz M. Periodontal plastic surgery for treatment of localized gingival recessions: A systematic review. J Clin Periodontol 2002;29(Suppl 3):178-194; discussion 195196.

29. Chambrone L, Lima LA, Pustiglioni FE, Chambrone LA. Systematic review of periodontal plastic surgery in the treatment of multiple recession-type defects. $J$ Can Dent Assoc 2009;75:203a-203f.

30. Chambrone L, Sukekava F, Araújo MG, Pustiglioni FE, Chambrone LA, Lima LA. Root coverage procedures for the treatment of localised recession-type defects. Cochrane Database Syst Rev 2009;(2):CD007161.

31. Klosek SK, Rungruang T. Anatomical study of the greater palatine artery and related structures of the palatal vault: Considerations for palate as the subepithelial connective tissue graft donor site. Surg Radiol Anat 2009;31:245-250.

32. Reiser GM, Bruno JF, Mahan PE, Larkin LH. The subepithelial connective tissue graft palatal donor site: Anatomic considerations for surgeons. Int J Periodontics Restorative Dent 1996;16:130-137.

33. Langer B, Langer L. Subepithelial connective tissue graft technique for root coverage. J Periodontol 1985; 56:715-720.

34. Harris RJ, Miller R, Miller LH, Harris C. Complications with surgical procedures utilizing connective tissue grafts: A follow-up of 500 consecutively treated cases. Int J Periodontics Restorative Dent 2005;25:449-459.

35. Greenstein G, Cavallaro J, Tarnow D. Practical application of anatomy for the dental implant surgeon. $J$ Periodontol 2008;79:1833-1846.

36. Monnet-Corti V, Santini A, Glise JM, et al. Connective tissue graft for gingival recession treatment: Assessment of the maximum graft dimensions at the palatal vault as a donor site. J Periodontol 2006;77:899-902.

37. Wang TM, Kuo KJ, Shih C, Ho LL, Liu JC. Assessment of the relative locations of the greater palatine foramen in adult Chinese skulls. Acta Anat (Basel) 1988;132: 182-186.

Correspondence: Professor Hom-Lay Wang, 1011 North University Ave., Ann Arbor, MI 48109-1078. Fax: 734/ 936-0374; e-mail: homlay@umich.edu

Submitted October 12, 2010; accepted for publication November 24, 2010. 